\title{
LOCALIZATION OF SODIUM CHANNELS IN CULTURED NEURAL CELLS $^{1}$
}

\author{
WILLIAM A. CATTERALL
}

Department of Pharmacology, University of Washington, Seattle, Washington 98195

\begin{abstract}
Sodium channels in cultured neural cells were localized by light microscopic autoradiography of specifically bound ${ }^{125} \mathrm{I}$-scorpion toxin. Ninety percent of the cell-bound ${ }^{125} \mathrm{I}$-scorpion toxin was associated specifically with sodium channels as assessed by the blocking of autoradiographic labeling by unlabeled scorpion toxin and by depolarization. Sodium channels were distributed uniformly in the surface membrane of neurites and cell bodies of both morphologically differentiated and undifferentiated cells of clone N18 of mouse neuroblastoma C1300. Sodium channels were distributed nonuniformly in many cultured spinal cord neurons. Visual observation indicated that $37 \pm 5 \%$ of cultured spinal cord neurons had a higher sodium channel density on the initial segment of one or more neurites than on the cell body. For these neurons, the density on one neurite initial segment averaged $7.4 \pm 1.9$-fold greater than on the adjacent cell body. This increased sodium channel density may be the basis of the lower threshold for action potential generation at the axon initial segment of motor neurons and some spinal interneurons in vivo.
\end{abstract}

Voltage-sensitive sodium channels play an essential role in action potential generation in nerve, skeletal muscle, and heart muscle. Physiological studies indicate a nonuniform distribution of electrical excitability in the cell surface membranes of each of these cell types. For example, in spinal motor neurons, the classical work of Eccles and colleagues (Coombs et al., 1955; Eccles, 1964) and Araki and Terzuolo (1962) showed that the initial segment of the axon has a lower threshold for action potential generation than the dendrites or cell body, leading to the conclusion that the action potential originates in the initial segment. This might result from a high density of sodium channels in the initial segment or from other localized changes in membrane properties. Anatomical techniques for localization of sodium channels would be valuable in assessing the role of a nonuniform distribution of sodium channels in specifying regional differences in electrical excitability in the cell surface membrane of spinal cord neurons and other excitable cells.

Sodium channels have three separate receptor sites for neurotoxins (for reviews, see Ritchie and Rogart, 1977; Catterall, 1980a). Neurotoxin receptor site 1 binds the heterocyclic guanidine derivatives, tetrodotoxin and saxitoxin, which block ion transport. Receptor site 2 binds grayanotoxin and the alkaloids, batrachotoxin, aconitine, and veratridine, which cause persistent activation of so-

\footnotetext{
This work was supported by Grants BNS 78-06870 and BNS 8021619 from the National Science Foundation.
}

dium channels. Receptor site 3 binds the polypeptides, scorpion toxin and sea anemone toxin, which slow inactivation of sodium channels and enhance persistent activation of sodium channels by alkaloid toxins. Of these three groups of toxins, only the polypeptides can be labeled to high specific activity with ${ }^{125} \mathrm{I}$ and fixed with aldehyde fixatives. These toxins, therefore, present the best opportunity for localization of sodium channels by autoradiography.

The binding of ${ }^{125}$ I-labeled scorpion toxin to sodium channels has been studied in detail (Catterall et al., 1976; Catterall, 1977). The binding is highly specific and is blocked by membrane depolarization or by unlabeled scorpion toxin. In this report, I describe the localization of specifically bound ${ }^{125}$ I-scorpion toxin on the cell surface of cultured mouse neuroblastoma cells and rat spinal cord neurons using autoradiographic methods.

\section{Materials and Methods}

Materials. Scorpion venom (Leiurus quinquestriatus) was purchased from Sigma. Scorpion toxin was purified, labeled with ${ }^{125} \mathrm{I}$ by lactoperoxidase-catalyzed iodination, and repurified as described previously (Catterall, 1977). Dulbecco's modification of Eagle's medium (DMEM), horse serum, newborn calf serum, and fetal calf serum were purchased from GIBCO; calf skin collagen was from Calbiochem; and plastic coverslips were from Lux.

Neuroblastoma cell cultures. Clone N18 of mouse neuroblastoma $\mathrm{C} 1300$ was used. For these studies, cells were subcultured with $0.02 \%$ trypsin as described previously (Catterall et al., 1976) and seeded at various dens- 
ities in $35-\mathrm{mm}$ Petri dishes containing a $25-\mathrm{mm}$ plastic coverslip. Cultures were maintained for up to 1 week in $2 \%(\mathrm{v} / \mathrm{v})$ newborn calf serum in DMEM and then were labeled and processed for autoradiography.

Rat spinal cord cell cultures. The method of Ransom et al. (1977) was used without modification except that DMEM was substituted for minimal essential medium (MEM). Plastic coverslips were coated with calf skin collagen in 35-mm Petri dishes and air dried. Spinal cords were dissected from 14-day-old rat embryos, minced with fine scissors, and dissociated in $1 \mathrm{ml}$ per spinal cord of DMEM containing $10 \%$ fetal calf serum and $10 \%$ horse serum by successive trituration using a Pasteur pipette of normal size followed by pipettes whose tip diameter was reduced by flaming. After each cycle of trituration, the tissue clumps were allowed to settle and the supernatants containing dissociated cells were removed and pooled. When further trituration did not release additional cells, the pooled supernatants were added to 35mm Petri dishes containing a collagen-coated coverslip and prewarmed, $\mathrm{CO}_{2}$-equilibrated DMEM with $10 \%$ fetal calf serum and $10 \%$ horse serum. Cell cultures were maintained in this medium until the background cells formed a confluent monolayer. Then, the medium was changed to DMEM with $10 \%$ horse serum plus $35 \mu \mathrm{g} / \mathrm{ml}$ of uridine and $15 \mu \mathrm{g} / \mathrm{ml}$ of $5^{\prime}$-fluoro- $2^{\prime}$-deoxyuridine to kill dividing non-neuronal cells. After 2 days, the medium was changed to DMEM with $10 \%$ horse serum for the remainder of the growth period.

In most experiments, spinal cord cells were seeded at a range of cell densities, and cultures having the largest number of well isolated neurons were used for study. Cultures were studied after 2 to 4 weeks of development in vitro. Similar labeling patterns were observed throughout this time period.

Autoradiographic labeling experiments. Both neuroblastoma cells and spinal cord neurons were labeled using the following procedure. Cell cultures were incubated for $60 \mathrm{~min}$ at $36^{\circ} \mathrm{C}$ with $1 \mathrm{~nm}$ mono $\left[{ }^{125} \mathrm{I}\right]$ iodo-scorpion toxin in standard binding medium consisting of $130 \mathrm{~mm}$ choline chloride, $5.4 \mathrm{mM} \mathrm{KCl}, 0.8 \mathrm{~mm} \mathrm{MgSO}_{4}, 5.5 \mathrm{~mm}$ glucose, and $50 \mathrm{mM}$ HEPES (4-(2-hydroxyethyl)-1-piperazineethanesulfonic acid, adjusted to $\mathrm{pH} 7.4$ with Tris (tris(hydroxymethyl) aminomethane) base). Cultures then were washed five times for $1 \mathrm{~min}$ each at $0^{\circ} \mathrm{C}$ with Dulbecco's phosphate-buffered saline and fixed by incubation for $16 \mathrm{hr}$ in $2.5 \%(\mathrm{w} / \mathrm{v})$ glutaraldehyde in phosphate-buffered saline. The fixative was washed away with ice cold $\mathrm{H}_{2} \mathrm{O}$ and the samples were dehydrated by washing twice in a series of ethanol solutions of increasing concentration $(30,50,70,95$, and $100 \%)$. The coverslips were coated with Kodak NTB-2 emulsion and exposed for 10 days to 2 weeks for spinal cord cells or 1 to 2 months for neuroblastoma cells. After development, the coverslips were mounted temporarily in $90 \%$ glycerol or permanently using Elvanol as a water-based mounting medium (Rodriguez and Dienhardt, 1960). The slides were examined by phase contrast, dark-field, and brightficld microscopy under oil immersion at magnifications ranging from $\times 250$ to $\times 1000$ using a Leitz Diavert microscope.

\section{Results}

Localization of sodium channels in neuroblastoma cells. The distribution of sodium channels on cultured neuroblastoma cells was studied first since the binding of ${ }_{1225} \mathrm{I}$-scorpion toxin to these cells has been analyzed thoroughly by biochemical methods (Catterall et al., 1976; Catterall, 1977). The toxin binds reversibly to a single class of specific receptor sites on the sodium channel with a $K_{D}$ of approximately $1 \mathrm{nM}$. The specific binding can be blocked completely by unlabeled scorpion toxin, unlabeled sea anemone toxin II, or depolarization (Catterall, 1977; Catterall and Beress, 1978; Couraud et al., 1978). Confluent cultures of N18 cells grown under standard culture conditions were incubated with $1 \mathrm{~nm}$ mono [ $\left.{ }^{12.5} I\right]$ iodo-scorpion toxin, washed, and processed for autoradiography as described under "Materials and Methods." Uniform labeling of the cells by ${ }^{125}$ I-scorpion toxin was observed. Labeling was reduced greatly by incubation in the presence of $135 \mathrm{~mm} \mathrm{~K} \mathrm{~K}^{+}$to depolarize the cells or by incubation in the presence of unlabeled scorpion toxin. To quantitate these findings, grain counts were made on cells assayed under control conditions or in the presence of $135 \mathrm{~mm} \mathrm{~K}^{+}$or $200 \mathrm{~nm}$ unlabeled scorpion toxin. The results of a typical experiment are presented in Table I. Grain counts were referred to the surface area of the upper surface of the cell by modeling the cell geometry as half of a prolate ellipsoid. This shape is a good approximation for nearly all of the cells grown under standard conditions. The results show that the density of autoradiographic grains was reduced $90 \%$ by incubation in the presence of unlabeled scorpion toxin and $88 \%$ by incubation under depolarizing conditions. Thus, approximately $90 \%$ of the labeling observed represents specific binding of the toxin to sodium channels.

Under standard growth conditions, most cells have small neurites. Visual inspection did not reveal any differences in grain density between cell bodies and neurites in these cultures. Growth of neuroblastoma cells in the presence of dibutyryl adenosine $3^{\prime}: 5^{\prime}$-monophosphate (but ${ }_{2}$ cAMP) causes marked morphological differentiation (Furmanski et al., 1971; Prasad and Hsie, 1971) and, in some cell lines, enhanced electrical excitability (Chalazonitis and Greene, 1974). It was of interest, therefore, to study N18. cells grown in the presence of $1 \mathrm{~mm}$ but $_{2}$ cAMP. Figure $1 A$ illustrates the appearance in the phase contrast microscope of a typical N18 cell grown under these conditions. After growth in but.cAMP, N18 cells were either flat and multipolar with several well developed neurites (Fig. 1, $A$ and $D$ ) or more rounded

TABLE I

Specificity of autoradiographic labeling of neuroblastoma cells

\begin{tabular}{clc} 
Cell Line & \multicolumn{1}{c}{$\begin{array}{c}\text { Incubation } \\
\text { Condition }\end{array}$} & Grain Density \\
\hline \multirow{2}{*}{$\mathrm{N} 18$} & & $\mu m^{-2}$ \\
& Control & $0.260 \pm 0.034$ \\
& $200 \mathrm{nM} \mathrm{ScTX}$ & $0.027 \pm 0.020$ \\
& $135 \mathrm{mM} \mathrm{KCl}$ & $0.030 \pm 0.013$ \\
\hline
\end{tabular}

"ScTX, unlabeled scorpion toxin. 

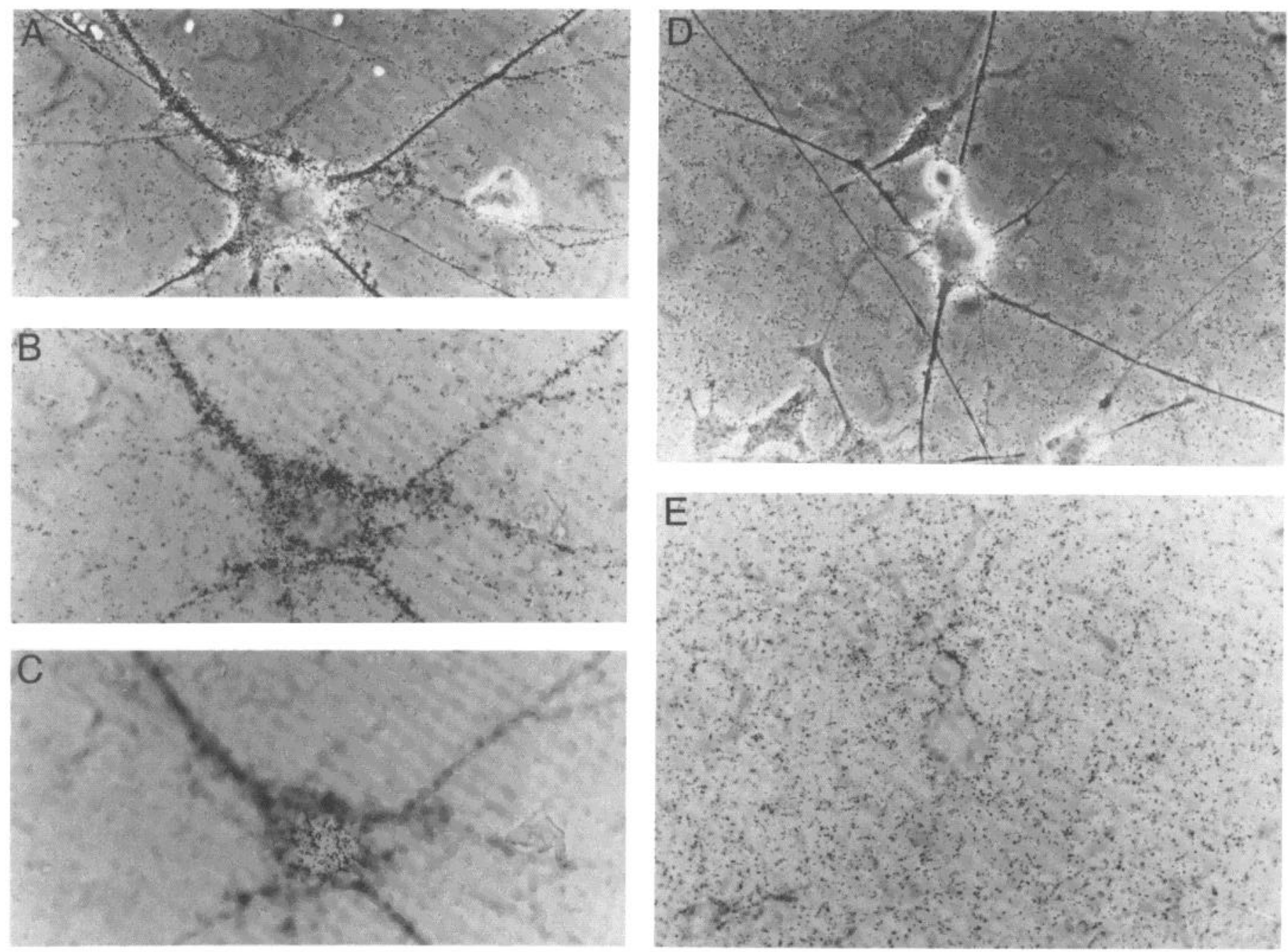

Figure 1. Localization of sodium channels in morphologically differentiated neuroblastoma cells. N18 cells were grown on plastic coverslips in $1 \mathrm{~mm}$ but 2 cAMP, labeled, and prepared for autoradiography as described under "Materials and Methods." Magnification $\times 312$. A, Phase contrast view of a cell labeled under specific labeling conditions. $B$, Bright-field view of the same cell as in $A$ focused in the plane of the neurites. $C$, Bright-field view of the same cell as in $A$ focused in the plane of the upper surface of the cell body. $D$, Phase contrast view of a different cell labeled in the presence of 200 nM unlabeled scorpion toxin. $E$, Bright-field view of the same cell as in $D$ focused in the plane of the neurites. No specific labeling of either neurites or cell body is observed on this or any other cell in this culture.

and bipolar (not shown). Autoradiographic analysis revealed substantial grain densities on both cell bodies and neurites (Fig. 1, $B$ and $C$ ). Incubation in the presence of unlabeled scorpion toxin completely prevented specific labeling (Fig. 1, $D$ and $E$ ) as did incubation in $135 \mathrm{~mm}$ $\mathrm{K}^{+}$(not shown). These results provide additional support for the conclusion that the labeling of sodium channels by scorpion toxin is specific.

Visual inspection revealed a uniform distribution of grains over cell bodies and neurites. To quantitate this observation, grain counts were made and referred to the surface of the cell body or neurite analyzed. Cell bodies were modeled as hemispheres or prolate hemi-ellipsoids, while the neurites were modeled as full cylinders. Focusing through the specimen at a magnification of $\times 1000$ confirmed that only grains on the upper surface of cell bodies were counted, whereas grains on all surfaces of
TABLE II

Grain density on differentiated neuroblastoma cells

\begin{tabular}{|c|c|c|c|c|}
\hline & $\begin{array}{l}\text { Average } \\
\text { Length }\end{array}$ & $\begin{array}{c}\text { Average } \\
\text { Width }\end{array}$ & $\begin{array}{c}\text { Grain } \\
\text { Density }^{a}\end{array}$ & Ratio \\
\hline & \multicolumn{2}{|c|}{$\mu m$} & $\mu m^{-2}$ & \\
\hline Cell body & 43.0 & 21.8 & $0.13 \pm 0.02$ & \\
\hline Neurite $^{b}$ & & 3.0 & $0.16 \pm 0.02$ & $1.2 \pm 0.2$ \\
\hline
\end{tabular}

" Surface area calculations were made as described in the text.

${ }^{b}$ A $50-\mu \mathrm{m}$ length of the largest neurite was counted and the average diameter was recorded.

the neurites (average diameter $=3 \mu \mathrm{m}$ ) were counted. The average values for dimensions and grain density of 10 randomly selected, isolated cells from the culture shown in Figure $1, A$ to $C$, are presented in Table II. The average ratio of grain density on the neurites to grain 
density on the cell body was $1.2 \pm 0.2$ (SEM, $n=10$ ). The ratio ranged from 2.2 to 0.5 for individual cells with the cell shown in Figure 1, $A$ to $C$, having a ratio of 1.7. Similar results were obtained with several other groups of cultures. Thus, within the resolution of these methods, sodium channels are distributed uniformly over the surface of cultured N18 neuroblastoma whether they are morphologically differentiated or undifferentiated.

Localization of sodium channels in cultured spinal cord neurons. In order to determine the distribution of sodium channels on normal neurons in cell culture, I prepared primary cultures of spinal cord neurons from 14-day rat embryos as described under "Materials and Methods." Neurons in similar cultures from mouse and chick have well developed electrical excitability due to the action of voltage-sensitive sodium and calcium channels (Ransom et al., 1977; Fischbach and Dichter, 1974; Ransom and Holz, 1977). After 2 to 4 weeks in culture, these cells were labeled with ${ }^{125} \mathrm{I}$-scorpion toxin and processed for autoradiography as described above for neuroblastoma cells. Neurons in the cultures were labeled, whereas non-neuronal cells were not (Fig. $2, A$ to $D$ ). Labeling was blocked completely by unlabeled scorpion toxin or by depolarization with $135 \mathrm{~mm} \mathrm{~K}^{+}$(not shown). Thus, scorpion toxin specifically labeled sodium channels in cultured spinal cord neurons and did not label nonneuronal cells.

In contrast to the results with neuroblastoma cells, the labeling of spinal cord neurons was often markedly nonuniform. The distribution of label on individual neurons was analyzed in sparse cell cultures since, in denser cultures, aggregation of cell bodies and formation of neurite fascicles prevented a careful study of grain densities on cell bodies and neurites. Two examples of cells with a strikingly nonuniform distribution of grains are presented in Figure 2, $A$ to $D$. The neuron illustrated in Figure 2, $A$ and $B$, is bipolar with a single large neurite leaving the cell body and traveling a long distance to the upper left and two smaller neurites leaving the cell body and traveling only a short distance to the upper right. The grain distribution over the cell body is sparse. A somewhat greater density is observed over the initial segment leading to the two smaller neurites, whereas a very high grain density is observed over the initial segment of the large neurite. The neuron illustrated in Figure 2, $C$ and $D$, has essentially the same arrangement of neurites. In this cell, both the cell body and smaller neurites leading to the left are sparsely labeled, whereas the initial segment of the long neurite leading to the right is more heavily labeled. In Figure 3, a cell with a nonuniform grain distribution is illustrated at higher magnification $(\times 1000)$. In panel $A$, the neurites are in the plane of focus, whereas panel $B$ focuses on the cell body. Again a greater density of grains over the initial segment of the principal neurite (on the left) is apparent. Strikingly nonuniform distributions of autoradiographic grains as illustrated for these cells were never observed in examination of several hundred labeled neuroblastoma cells and therefore are unlikely to be artifacts generated by the procedures for labeling and processing the specimens.

To estimate the generality of this nonuniform labeling
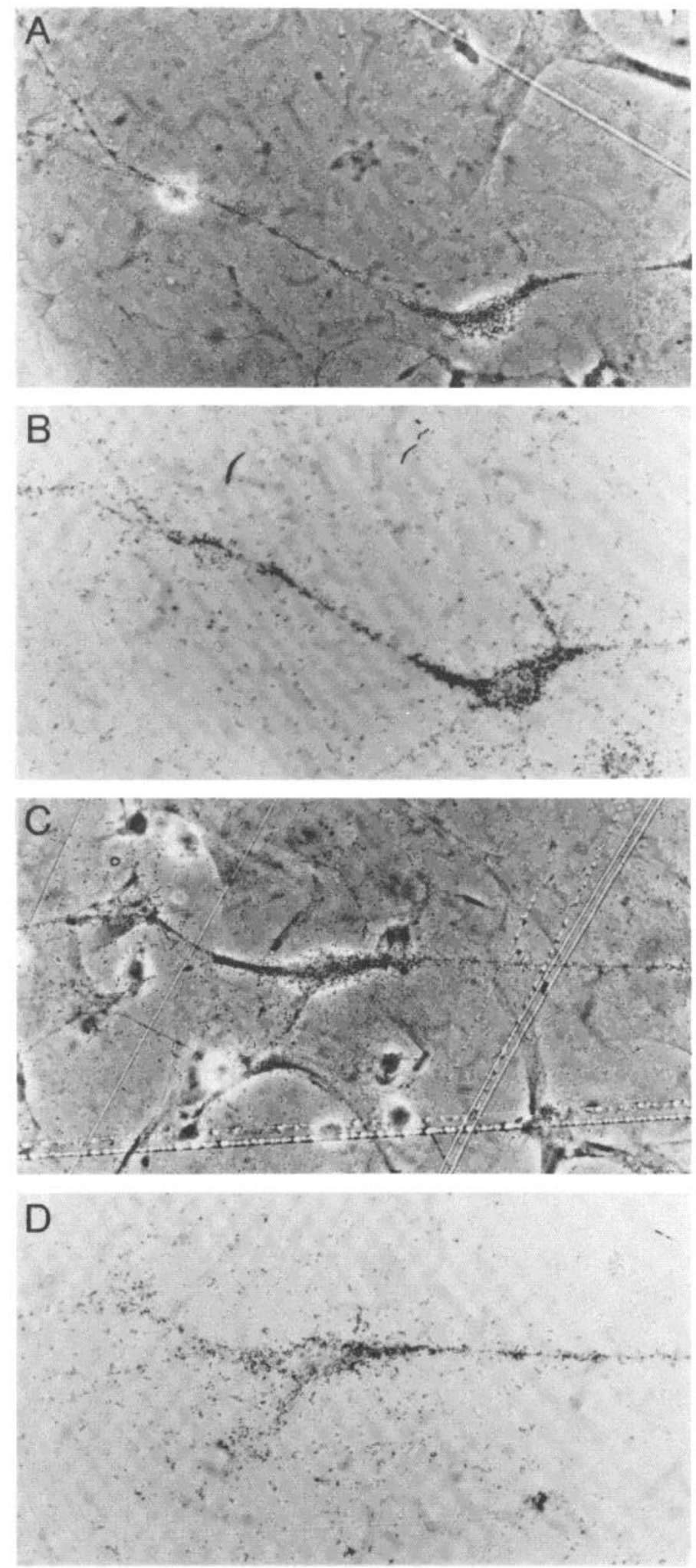

Figure 2. Localization of sodium channels in cultured spinal cord neurons. Spinal cord neurons were grown, labeled, and prepared for autoradiography as described under "Materials and Methods." Magnification $\times 312$. A, Phase contrast view of a cell labeled under specific labeling conditions. $B$, Bright-field view of the same cell as in $A$. $C$, Phase contrast view of a second cell labeled under specific labeling conditions. $D$, Bright-field view of the same cell as in $C$. 

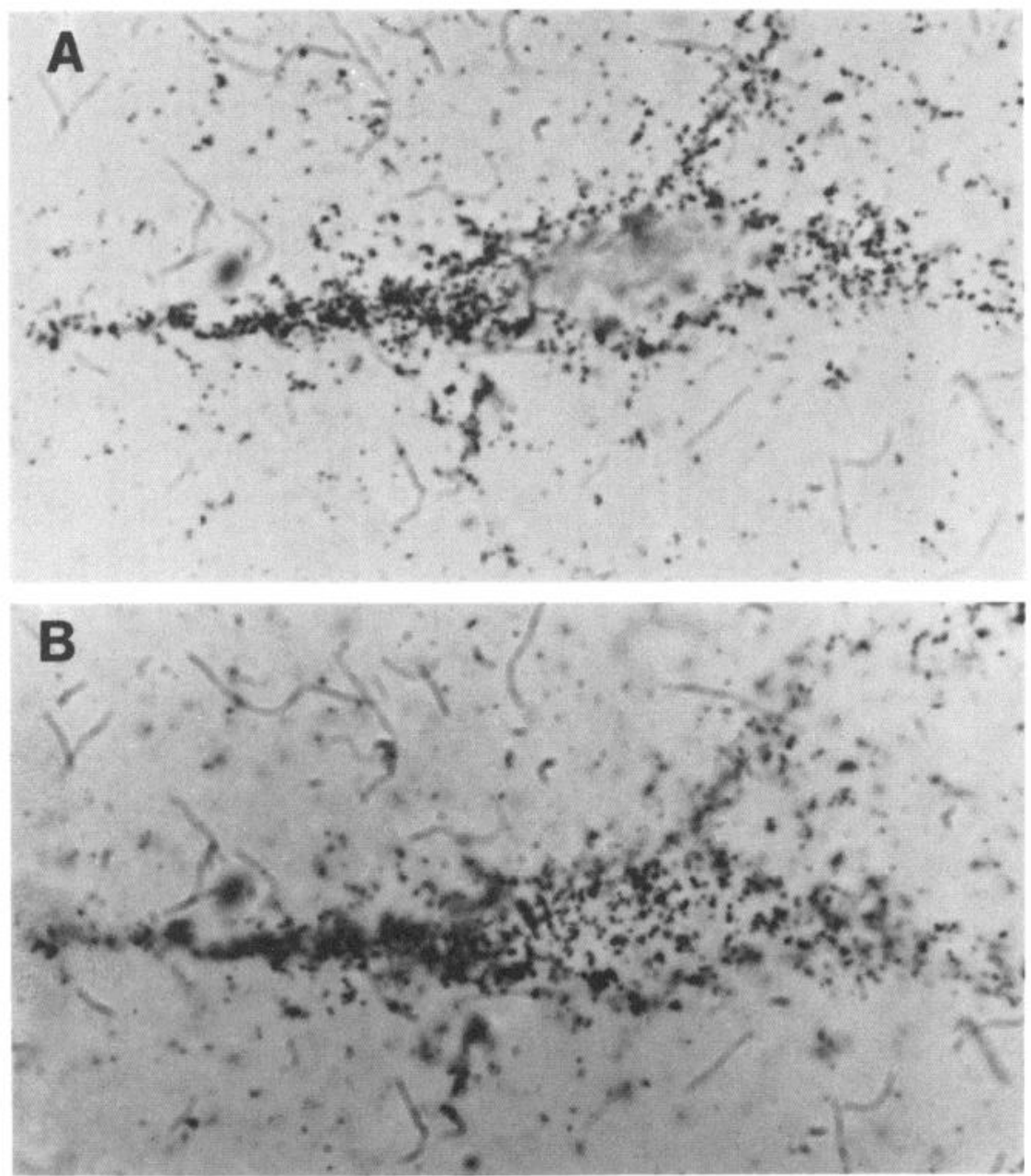

Figure 3. Localization of sodium channels in cultured spinal cord neurons. Magnification $\times 1000$. $A$, Bright-field view of a cell labeled under specific labeling conditions with the plane of focus at the level of neurites. $B$, Brightfield view of the same cell with the plane of focus at the level of the surface of the cell body.

pattern, sparse cultures were scanned and isolated neurons were scored visually for the presence or absence of a nonuniform distribution of grains. In several cultures, $37 \pm 5 \%$ of the cells examined had one neurite initial segment with a grain density obviously greater than that on the cell body. Less than $2 \%$ of the cells had a greater grain density on the cell body than on all neurites. Thus, a substantial fraction of the individual neurons examined had at least one neurite whose initial segment had a grain density higher than the cell body, indicating a specific localization of sodium channels in this region of the cell surface. The grain density over more distal regions of the neurites was not very consistent. In some cells (Fig. 2, $A$ and $C$ ), the density of grains at distal points on the neurites diminished to that at the cell body, whereas in others, the major neurite(s) was densely labeled uniformly.

To provide a quantitative estimate of the extent of the nonuniformity in grain density in the cells in which an uneven distribution was visually apparent, several typical cells having a simple cell geometry were analyzed as described for neuroblastoma cells above. The average
TABLE III

Grain density on spinal cord neurons

\begin{tabular}{|c|c|c|c|c|}
\hline & $\begin{array}{c}\text { Average } \\
\text { Length }\end{array}$ & $\begin{array}{l}\text { Average } \\
\text { Width }\end{array}$ & $\begin{array}{c}\text { Grain } \\
\text { Density }^{a}\end{array}$ & Ratio \\
\hline & \multicolumn{2}{|c|}{$\mu m$} & $\mu m^{-2}$ & \\
\hline Cell body & 26.0 & 16.6 & $0.19 \pm 0.03$ & $7.4 \pm 1.9$ \\
\hline Neurite ${ }^{b}$ & $36.0^{h}$ & 1.7 & $1.37 \pm 0.27$ & \\
\hline
\end{tabular}

"Surface area was calculated as described in the text.

${ }^{b}$ The length of the region of high grain density and its average diameter are presented.

dimensions and grain density for one representative group of cells is presented in Table III. For these cells, the density over the initial segment of the largest neurite was $1.4 \pm 0.3$ grains $/ \mu \mathrm{m}^{2}$, while the density over the cell body was $0.19 \pm 0.03$ grains $/ \mu \mathrm{m}^{2}$. Therefore, the ratio of grain densities for these cells was 7.4. This is likely to be an underestimate of the true density ratio since the labeling of neurite initial segments was usually too dense to allow a precise count of all grains. Thus, it is likely that $37 \%$ of the cells in the population have at least a 7 - 
fold greater density of sodium channels on the initial segment of their largest neurite than on the cell body.

\section{Discussion}

The results described demonstrate the utility of ${ }^{125} \mathrm{I}$ scorpion toxin autoradiography for the localization of sodium channels in cultured neurons. The specificity of labeling is supported by three lines of evidence: block of specific labeling by unlabeled scorpion toxin, block of labeling by depolarization, and lack of labeling of nonneuronal cells in the primary spinal cord cultures. The method is quite sensitive. Biochemical experiments have shown that undifferentiated neuroblastoma cells have 25 scorpion toxin receptor sites per $\mu \mathrm{m}^{2}$ of cell surface (Catterall, 1977). This low density of sites gives specific labeling 9 times greater than nonspecific labeling of cells. The method will be useful, therefore, for most neuronal cells in culture.

The method may not be generally applicable, however. Cultured skeletal muscle cells from chick (Catterall, $1980 \mathrm{~b}$ ) and rat (Lawrence and Catterall, 1981) have sodium channels with relatively low affinity for scorpion toxin, and rat skeletal muscle cells are not specifically labeled by ${ }^{12: 5}$ I-scorpion toxin (W. A. Catterall, unpublished results). Cultured rat heart muscle cells have low affinity for scorpion toxin (W. A. Catterall, unpublished results), whereas, in cultured chick heart cells, the ratio of specific to nonspecific binding of ${ }^{125} \mathrm{I}$-scorpion toxin is low even though the $K_{I}$ for toxin is in the same range as for neural cells (Couraud et al., 1980). Scorpion toxin may not be a useful tool to localize sodium channels in cultured muscle cells.

Different patterns of localization of sodium channels were observed in the two cell types studied. In N18 cells, sodium channels were distributed uniformly within the resolution of the methods used. In contrast, sodium channels were localized in high density in initial segments of individual neurites of many cultured spinal cord neurons. Although the number and geometry of neurites on spinal cord neurons was highly variable, it was typical of these cells to have only one or two neurites highly labeled by scorpion toxin. The high density of sodium channels typically began near the cell body and continued a variable distance along the neurite, sometimes extending the full length of the neurite. These results show that cultured spinal cord neurons have the capacity to restrict the location of sodium channels in their cell surface. Localized regions of high density of sodium channels would be expected to have important physiological consequences in initiation and propagation of action potentials.

The classical studies of Eccles and colleagues (Coombs et al., 1955; Eccles, 1964) and Araki and Terzuolo (1962) showed that the threshold depolarization required for initiation of an action potential in spinal motor neurons was 2 to 3 times greater at the cell body than at the initial segment of the axon. Subsequent electrophysiological studies have shown that this is a common property of vertebrate central neurons (reviewed by Eccles, 1964). Some classes of spinal interneurons have a threshold difference between the initial segment and cell body, while other do not. Goldfish Mauthner neurons and both cortical and hippocampal pyramidal neurons also have a substantial threshold difference between these two membrane regions.

These differences in threshold for action potential generation could arise from differences in passive membrane properties, in the time and voltage dependence of sodium channel activation, or in the density of sodium channels in the surface membrane of the cell body and axon initial segment. The results presented here show that many cultured spinal cord neurons have a 7 -fold higher density of sodium channels on one or more neurite initial segments than on the cell body. This property of cultured spinal neurons may be the mechanism responsible for the difference in threshold of the cell body and axon initial segment observed in motor neurons and spinal interneurons in vivo. A greater density of sodium channels would reduce the threshold for action potential generation by reducing the fraction of sodium channels that must be activated to give an all-or-none response. At any given depolarization, a larger number of sodium channels would be activated and therefore, an action potential could be initiated at more negative membrane potentials. This mechanism provides a plausible explanation for the physiological results. It will be of considerable interest to determine whether spinal motor neurons have a similar distribution of sodium channels in vivo.

\section{References}

Araki, T., and C. A. Terzuolo (1962) Membrane currents in spinal motoneurons associated with the action potential and synaptic activity. J. Neurophysiol. 25: 772-789.

Catterall, W. A. (1977) Membrane potential dependent binding of scorpion toxin to the action potential sodium ionophore. Studies with a derivative prepared by lactoperoxidase-catalyzed iodination. J. Biol. Chem. 252: 8660-8668.

Catterall, W. A. (1980a) Neurotoxins that act on voltage-sensitive sodium channels in excitable membranes. Annu. Rev. Pharmacol. Toxicol. 20: 15-43.

Catterall, W. A. (1980b) Pharmacologic properties of voltagesensitive sodium channels in chick muscle fibers developing in vitro. Dev. Biol. 78: 222-230.

Catterall, W. A., and L. Beress (1978) Sea anemone toxin and scorpion toxin share a common receptor site on the action potential sodium ionophore. J. Biol. Chem. 253: 7393-7396.

Catterall, W. A., R. Ray, and C. S. Morrow (1976) Membrane potential dependent binding of scorpion toxin to the action potential sodium ionophore. Proc. Natl. Acad. Sci. U. S. A. 73: $2682-2686$.

Chalazonitis, A., and L. Greene (1974) Enhancement in excitability properties of mouse neuroblastoma cells cultured in presence of dibutyryl cyclic AMP. Brain Res. 72: 340-345.

Coombs, J. S., J. C. Eccles, and P. Fatt (1955) The electrical properties of the motoneurone membrane. J. Physiol. (Lond.) 130: 291-325.

Couraud, F., H. Rochat, and S. Lissitzky (1978) Binding of scorpion and sea anemone neurotoxins to a common site related to the action potential $\mathrm{Na}^{+}$ionophore in neuroblastoma cells. Biochem. Biophys. Res. Commun. 83: 1525-1530.

Couraud, F., H. Rochat, and S. Lissitzky (1980) Binding of scorpion neurotoxins to chick embryonic heart cells in culture and relationship to calcium uptake and membrane potential. Biochemistry 19: 457-462.

Eccles, J. C. (1964) Excitatory responses of spinal neurons. Prog. Brain Res. 12: 1-31. 
Fischbach, G. D., and M. A. Dichter (1974) Electrophysiologic and morphologic properties of neurons in dissociated chick spinal cord cultures. Dev. Biol. 37: 100-116.

Furmanski, P., D. J. Silverman, and M. Lubin (1971) Expression of differentiated functions in mouse neuroblastoma mediated by dibutyryl cyclic AMP. Nature 233: 413-415.

Lawrence, J. C., and W. A. Catterall (1981) Tetrodotoxin-insensitive sodium channels in cultured rat muscle cells. Ion flux studies of neurotoxin action. J. Biol. Chem. 256: 6213-6222.

Prasad, K. N., and A. W. Hsie (1971) Morphological differentiation of mouse neuroblastoma cells induced in vitro by dibutyryl cyclic AMP. Nature New Biol. 233: 141-143.

Ransom, B. R., and R. W. Holz (1977) Ionic determinants of excitability in cultured mouse dorsal root ganglion and spinal cord cells. Brain Res, 136: 445-453.

Ransom, B. R., E. Neale, M. Henkart, P. N. Bullock, and P. G. Nelson (1977) Mouse spinal cord in cell culture. I. Morphology and intrinsic neuronal electrophysiologic properties. J. Neurophysiol. 40: 1132-1150.

Ritchie, J. M., and R. B. Rogart (1977) The binding of saxitoxin and tetrodotoxin to nerve membranes. Rev. Physiol. Biochem. Pharmacol. 79: 1-51.

Rodriguez, J., and F. Dienhardt (1960) Preparation of a semipermanent mounting medium for fluorescent antibody studies. Virology 12: 316-317. 